\title{
CIRCUITO ONLINE COMO PROPOSTA PARA O ENSINO DE MICROBIOLOGIA
}

\section{ONLINE CIRCUIT AS PROPOSAL TEACHING OF MICROBIOLOGY}

\author{
CIRCUITO ONLINE COMO PROPUESTA PARA \\ LA ENSEÑANZA DE LA MICROBIOLOGÍA
}

\author{
Laiane Oliveira Lima Soares ${ }^{1}$; Lucas Lopes de Sousa ${ }^{2}$; Luis Costa Coutinho ${ }^{3}$; \\ Francimar Batista Sousa ${ }^{4}$; Natália Rodrigues da Silva ${ }^{5}$; Iara Gabriela dos Santos \\ Nascimento ${ }^{6}$; Marcos Antônio Lima Mário ${ }^{7}$; Marlúcia da Silva Bezerra Lacerda ${ }^{8}$
}

\begin{abstract}
Resumo
O presente estudo objetivou apresentar uma ferramenta para auxiliar, de forma online, o ensino prático de microbiologia, contribuindo para um aprendizado mais efetivo dos conceitos relacionados a grande área das ciências biológicas. A pesquisa do tipo exploratória e de abordagem quali-quantitativa, foi balizada pelo planejamento, organização e realização do circuito online de microbiologia (COM), fazendo uso de metodologias ativas para implementação da proposta. Todas as etapas da pesquisa foram realizadas por discentes regularmente matriculados no curso de licenciatura em ciências biológicas do Instituto Federal do Piauí. Consideramos que o COM despertou interesse nos estudantes por ter caráter dinâmico e pouco convencional, fugindo das propostas tradicionais de ensino, e contribuindo, de forma mais consistente para o aprendizado na área da microbiologia.
\end{abstract}

Palavras-chave: Rotação por estação; Metodologias ativas; Ensino de Microbiologia; Aprendizagem.

${ }^{1}$ Enfermeira graduada - Centro Universitário UNINOVAFAPI. Teresina, PI - Brasil. Discente do curso de Licenciatura em Biologia pelo Instituto Federal do Piauí (IFPI). Teresina, PI - Brasil. E-mail: Laianne_oliveira@hotmail.com

${ }^{2}$ Graduado em Engenharia Agronômica - Universidade Estadual do Piauí (UESPI). Teresina, PI - Brasil. Graduando em Licenciatura em Ciências Biológicas - Instituto Federal do Piauí (IFPI).Teresina, PI - Brasil. Email: lucaslopesdesousa1994@gmail.com

${ }^{3}$ Biomédico - Centro Universitário UNINOVAFAPI. Teresina, PI - Brasil. Licenciando em Ciências Biológicas - Instituto Federal de Educação, Ciências e Tecnologia do Piauí (IFPI). Teresina, PI - Brasil. E-mail: luisscosta10@gmail.com

${ }^{4}$ Graduando em Ciências - Instituto Federal de Ciência e Tecnologia do Piauí (IFPI) Campus Teresina Central. Teresina, PI - Brasil. E-mail: batistafrancimar36@gmail.com

${ }^{5}$ Graduanda de Licenciatura em Ciências Biológicas - Instituto Federal de Educação, Ciências e Tecnologia do Piauí (IFPI) Teresina, PI - Brasil. E-mail: nataliarodriguesdasilva22@ gmail.com

${ }^{6}$ Graduanda em Ciências Biológicas - Instituto Federal de Ciência e Tecnologia do Piauí (IFPI) Campus Teresina Central. Teresina, PI - Brasil. E-mail: Iaragabriela00801@gmail.com

${ }^{7}$ Licenciando em Ciências Biológicas - Instituto Federal de Ciência e Tecnologia do Piauí (IFPI) Campus Teresina Central. Tereseina, PI - Brasil. E-mail: marcozlima22@gmail.com

${ }^{8}$ Doutora em Ciência Animal - Universidade Federal do Piauí (UFPI). Teresina, PI - Brasil. Docente do Curso de Licenciatura em Ciências Biológicas - Instituto Federal do Piauí (IFPI) Campus Teresina Central. Teresina, PI - Brasil. E-mail: marlucia.lacerda@ifpi.edu.br 


\begin{abstract}
This study aimed to present a tool to assist, online, the practical teaching of microbiology, contributing to a more effective learning of concepts related to the large area of biological sciences. The exploratory research, with a quali-quantitative approach, was guided by the planning, organization and realization of the online microbiology circuit (COM), making use of active methodologies to implement the proposal. All stages of the research were carried out by students regularly enrolled in the degree course in biological sciences at the Federal Institute of Piauí. We believe that COM aroused interest in students for having a dynamic and unconventional character, fleeing from traditional teaching proposals, and contributing more consistently to learning in the area of microbiology.
\end{abstract}

Keywords: Rotation by Station; Active Methodologies; Teaching of Microbiology; Learning.

\title{
Resumen
}

Este estudio tuvo como objetivo presentar una herramienta para ayudar, en línea, a la enseñanza práctica de la microbiología, contribuyendo a un aprendizaje más efectivo de conceptos relacionados con el gran área de las ciencias biológicas. La investigación exploratoria, con enfoque cuali-cuantitativo, estuvo guiada por la planificación, organización y realización del circuito de microbiología en línea (COM), haciendo uso de metodologías activas para implementar la propuesta. Todas las etapas de la investigación fueron realizadas por estudiantes matriculados regularmente en la carrera de ciencias biológicas en el Instituto Federal de Piauí. Creemos que COM despertó el interés de los estudiantes por tener un carácter dinámico y poco convencional, huyendo de las propuestas didácticas tradicionales, y contribuyendo de manera más consistente al aprendizaje en el área de la microbiología..

Palabras clave: Rotación por estación; Metodologias ativas; Ensino de Microbiologia; Aprendizagem.

$$
* * *
$$

\section{Introdução}

A microbiologia, enquanto subárea da biologia, caracteriza-se como fundamental para a compreensão de conceitos relacionados à educação em saúde. Muitos estudos relacionados a esta ciência são de grande importância para a compreensão do mundo e de como acontecem as doenças, a exemplo, temos a pesquisa do cientista holandês Antony Van Leeuwenhoek que, ainda no séc. XVII, fazendo uso de microscópio bem rudimentar, observou a presença dos microrganismos, resultando em uma grande contribuição para a microbiologia. No Séc. XXI, os estudos da microbiologia continuam a contribuir, não só com promoção de uma sociedade mais protegida dos "micróbios" que provocam enfermidades, mas também capaz de reconhecer e valorizar os tantos benefícios que o conhecimento sobre a vida microbiana nos traz.

Nesse contexto, ao final do ano de 2019, o mundo foi impactado enormemente com a chegada e descoberta de um novo coronavírus, o SARS-CoV-2, associado com a doença Covid19, chegando em setembro de 2021 ao número de 4.592.934 mortes notificadas a nível global, com as Américas sendo detentoras do maior número de óbitos entre os continentes e o Brasil como o terceiro colocado neste triste ranking de mortes pela covid-19 com um total de 584,2 pessoas falecidas, perdendo apenas para os Estados Unidos e Índia (OMS, 2021). Este crescente número de casos de mortes fez o mundo planejar estratégias de segurança com restrições sanitárias para conter a transmissibilidade, entre elas o distanciamento e isolamento social que levaram ao 
DOI: $10.46667 /$ renbio.v $14 \mathrm{i} 2.508$

fechamento das unidades escolares e a adoção do ensino remoto com propostas pedagógicas online.

Nesse sentido, no Brasil admitiu-se formalmente as atividades pedagógicas não presenciais, a partir de um parecer do Conselho Nacional de Educação (CNE/CP n ${ }^{\circ}$ 5/2020, aprovado em 28 de abril de 2020). Este Parecer surge orientando para a reorganização do calendário escolar e o cômputo de atividades não presenciais em razão da Pandemia da COVID19, tendo como caminho o ensino remoto emergencial e a possibilidade de utilização das Tecnologias Digitais da Informação e Comunicação como alternativa para criar formas de diminuição das desigualdades de aprendizado (BRASIL, 2020).

Posto isso, foi planejado, desenvolvido e executado por um docente e um grupo de estudantes da Licenciatura em Ciências Biológicas do Instituto Federal do Piauí, regularmente matriculados no componente curricular de Projeto Integrador III, uma proposta de ensino/aprendizagem denominado Circuito Online de Microbiologia (COM), aplicado de forma remota em sua totalidade. Neste trabalho, objetivou-se contribuir com a disponibilização de uma ferramenta pedagógica capaz de auxiliar outros estudantes, em especial os do mesmo curso e instituição matriculados na componente curricular de microbiologia, criando uma possibilidade de aprendizagem mais efetiva de conteúdos teóricos e práticos.

Contudo, a proposta pedagógica em destaque nesta pesquisa, o circuito online, tem viabilidade de aplicação em diferentes contextos de ensino de biologia, desde que as atividades sejam adequadas à subárea requisitada, seja ela botânica, zoologia, parasitologia, ecologia, entre outras. Há ainda de se destacar a contribuição deste estudo com a formação inicial de futuros professores de biologia para a educação básica brasileira, tornando-os mais capacitados para enfrentar as salas de aulas no contexto à distância.

\section{Embasamento teórico}

Nos tempos atuais, mais do que nunca, o professor precisa desenvolver maneiras de tornar o processo de ensino e aprendizagem significativo, buscando alternativas didáticas para que os alunos possam construir um conhecimento que tenha significado, o que irá possibilitar uma aprendizagem mais efetiva e se contrapor ao processo apenas mecânico ou de pura memorização, que pouco contribui, servindo apenas para responder uma avaliação (BERNARDI et al., 2019).

Uma das finalidades do ensino da ciência é garantir que os estudantes compreendam o funcionamento e a construção dos fenômenos naturais. Nesse intuito de facilitar a aprendizagem dos estudantes, os professores utilizam diferentes recursos metodológicos para garantir o processo de ensino-aprendizagem dos alunos na construção de seus conhecimentos e na sensibilização de questões sociais (SCHEID et al., 2007; SARMENTO et al., 2017).

Neste sentido, justifica-se que Organização Mundial de Saúde (OMS) recomenda estratégias como medidas de prevenção de infecções para a evitar a proliferação de 
DOI: $10.46667 /$ renbio.v14i2.508

microrganismos no ambiente. Com a constatação de que microrganismos existiam e afetavam os seres humanos, percebeu-se a necessidade de adotar medidas de higiene e políticas públicas de saúde que, a longo prazo, duplicariam a nossa expectativa de vida. Hoje ainda morrem cerca de 20 milhões de pessoas por ano vítimas de infecções. Mas, a vacinação em massa já erradicou doenças como a varíola e diminuiu o número de casos de tuberculose, raiva e outros males.

Assim, tendo em vista que os conteúdos de microbiologia estão em geral focados para estes seres como apenas causadores de doenças e ainda com conteúdos restritamente teóricos, os alunos acabam desenvolvendo uma abstração muito forte na temática. Por isso a prática em microbiologia faz-se importante para que os estudantes consigam desenvolver processos cognitivos que facilitam a clareza do assunto, fazendo com que o aluno seja proativo no processo de aprendizagem (AZEVEDO; SODRÉ, 2014).

Em síntese, a microbiologia deixou de ser tema apenas restrito às salas de aula de ensino superior ou a laboratórios de pesquisa para ser tema relacionado às questões básicas de cidadania, envolvendo o meio ambiente, a higiene, a política e entre outros (PRADO; TEODORO; KHOURI, 2020).

\section{Procedimentos metodológicos}

Este estudo trata-se de uma pesquisa exploratória, a qual segundo Gil (2002), tem como objetividade proporcionar maior familiaridade com o problema, com vistas a torná-lo mais explícito ou a constituir hipóteses, onde se tem como objeto principal o aprimoramento de ideias ou descoberta de intuições. Junto a isso, se empregou uma abordagem quali-quantitativa utilizando de plataformas virtuais para apresentar uma proposta pedagógica de ensino/aprendizagem de microbiologia no sentido de auxiliar a aprendizagem de conteúdos, práticos e teóricos, no ensino remoto desta componente curricular.

O foco amostral foi formado por estudantes voluntários regularmente matriculados nos Módulos 1 e 3 do Curso de Licenciatura em Ciências Biológicas do Instituto Federal do Piauí, uma instituição de ensino superior alocada no município de Teresina, capital do estado do Piauí. A pesquisa fez parte de estratégia metodológica apresentada na componente curricular Projeto Integrador III, desenvolvida no período letivo de 2020.1.

A divulgação do Circuito Online de Microbiologia, a partir de agora representado pela sigla COM, entre os estudantes foi realizada através de três mecanismos principais de acesso livre e gratuito: $i$ - As redes sociais como Whatsapp, Instagram e Facebook que são de maior abrangência e alcance entre os estudantes; ii- Pela plataforma Google Classroom, atreladas aos grupos de estudos dos estudantes; iii- Por mensagens compartilhadas no mural as salas virtuais das disciplinas de Introdução ao Laboratório de Biologia (ILB), Microbiologia (MB) e Projeto Integrador III (PI III), dos Módulos 1, 3 e 3, respectivamente. Para avaliar qual destas vias de 
DOI: $10.46667 /$ renbio.v14i2.508

divulgação melhor funcionou no contexto desta pesquisa os participantes foram também consultados sobre a via de acesso pela qual tiveram conhecimento do COM.

A proposta pedagógica aqui apresentada foi o projeto de uma das equipes de PI III e consistiu em agrupar Atividades Didáticas Teóricas e Práticas (ADTP) num formato de circuito (Circuito Online de Microbiologia), onde os voluntários participantes poderiam oportunamente fazer uso de conceitos relacionados à Microbiologia dentro de um protocolo preparado exclusivamente para este estudo (Figura 01). O circuito foi apresentado dentro de um conceito de Rotação por Estação, com os alunos percorrendo remotamente cada atividade proposta, de forma interativa, intuitiva e autoguiada.

Figura 01: Atividades Didáticas Teóricas e Práticas (ADTP) desenvolvidas no Circuito Online de Microbiologia.

\begin{tabular}{|c|c|}
\hline $\begin{array}{c}\text { ATIVIDADES PRÁTICAS } \\
\text { DESENVOLVIDAS }\end{array}$ & DESCRIÇÃO \\
\hline ADTP 1 - Microscopia & História e Evolução dos Microscópio [1] [2] \\
\hline ADTP 2 - Coloração de Gram & $\begin{array}{c}\text { Técnica de Coloração diferencial de Gram } \\
\text { para visualização, caracterização e } \\
\text { classificação de bactérias }\end{array}$ \\
\hline ADTP 3 - Higienização das mãos & $\begin{array}{c}\text { Demonstração do passo a passo da técnica } \\
\text { para higienização correta das mãos (água e } \\
\text { álcool gel) }\end{array}$ \\
\hline
\end{tabular}

Fonte: Dos Próprios Autores.

As estações do COM, foram alocadas numa turma com a mesma denominação na plataforma Google Classroom estruturada apenas para este fim, onde os participantes voluntários tiveram acesso por meio do recebimento de um código de ingresso disponibilizado pelos autores a todos os estudantes das turmas de ILB, MB e PI III que manifestaram interesse. Dessa forma, todas as atividades do circuito foram postadas nessa plataforma, podendo ser acessada de forma assíncrona em qualquer momento a critério do voluntário, dentro de período pré-estabelecido para realização do circuito, que foi de 7 (sete) dias corridos.

\section{Resultados e discussões}

Durante a realização Circuito Online de Microbiologia (COM) foram propostas 3 (três) Atividades Didáticas Teóricas e Práticas (ADTP) já apresentadas no Quadro 01 e que abordaram conteúdos considerados fundamentais na microbiologia. 
DOI: $10.46667 /$ renbio.v $14 \mathrm{i} 2.508$

Na ADTP 1 destacou-se o microscópio, que é um dos instrumentos mais usados em laboratórios de microbiologia, devido ao fato de ser obrigatório e indispensável quando se quer realizar observações mais detalhadas dos microrganismos. Vale destacar especialmente o microscópio óptico que é o mais acessível deles, por ser mais barato e de fácil manuseio. Fez parte ainda deste contexto da microscopia e não foi possível deixar de fora a história deste equipamento e as categorias eletrônicas que com a evolução no poder de ampliação e resolução possibilitou ao mundo visualizar muito além do que os olhos humanos poderiam. A trajetória histórica do microscópio confunde-se e anda paralelamente a história da biologia e as grandes descobertas nas suas outras subáreas, como a citologia, a zoologia, a parasitologia e a genética, por exemplo.

Esta atividade (ADTP) do circuito foi descrita em dois momentos: $1^{\circ}$ ) relacionado a visualização de um vídeo autoral sobre introdução a microscopia; $2^{\circ}$ ) de participação em um Quiz na plataforma digital Kahoot, através de um link previamente disponibilizado (https://kahoot.it/share/introducao-a-microscopia) para a turma de voluntários participantes do circuito. O Quis consistiu em um jogo formado por testes de múltipla escolha sobre o conteúdo proposto e os estudantes teriam que responder de forma correta e em um tempo padrão. Se sairia melhor no jogo quem respondesse correto e em menor tempo à sequência de perguntas.

"O Kahoot é uma plataforma de aprendizagem gratuita baseada em jogos que tem como missão institucional descrita em seu site como "desbloquear o potencial mais profundo de cada aluno de todas as idades e em todos os contextos, através de um aprendizado divertido, mágico, inclusivo e envolvente" (KAHOOT, 2021).

Esse ambiente virtual possibilita a criação de questionários e discussões que podem ser respondidas por usuários que estejam conectados à Internet por meio de smartphones, tablet ou computadores. Desse modo, a ideia principal do Kahoot é ser uma plataforma onde o professor e os alunos podem interagir, em sala de aula presencial ou virtual, simulando um jogo de conhecimento competitivo. A motivação é envolver os alunos através da transformação da sala de aula em um game show, onde o professor seria o apresentador e todos os alunos podem competir ganhando pontos através de respostas corretas em várias questões relacionadas ao assunto que está sendo ensinado na aula (WANG, 2015).

A ADTP 2 permitiu aos estudantes voluntários a visualização de bactérias pelo método de coloração de Gram. Esta proposta foi realizada por meio da disponibilização de uma videoaula gravada pelos pesquisadores, com objetivo de explicar com detalhes a realização da técnica de coloração diferencial de bactérias pelo método de Gram, demonstrando o passo a passo dessa prática laboratorial de grande importância para a microbiologia. Associado aos vídeo, foi disponibilizado um link para acesso a um laboratório virtual de microbiologia, disponível gratuitamente pelo link: https://www.biomedicinapadrao.com.br/2014/12/teste-seuconhecimento-e-sua-pratica-no.html onde os estudantes puderam ter a possibilidade de treinar a realização do procedimento da coloração de gram de forma online e gratuita. 
DOI: $10.46667 /$ renbio.v 14 i 2.508

Entende-se que conhecer o passo a passo da técnica de coloração de Gram e os conhecimentos relacionados à aplicação prática cotidiana dessa técnica será de grande importância para os estudantes, pois consideramos que não se concebe um graduado em Ciências Biológicas que não compreenda a importância de tal procedimento. Contudo, ainda destacamos que o momento de ensino remoto emergencial não permitiria a realização da prática de forma presencial no laboratório de microbiologia, assim, supõe-se que esta prática online e a simulação do passo a passo em um laboratório virtual, usado para tornar a assimilação deste conteúdo de uma forma mais fácil, prática e dinâmica e menos imaginária e teórica.

Na ADTP 3 foi proposto a higienização correta das mãos. Esta proposta correspondeu à atividade que mais pode ser relacionada ao momento de pandemia em que todos estão expostos sendo, portanto, a que teve mais significado para os voluntários depois da ADTP 2. Esta prática foi desenvolvida através de uma vídeo aula com a ilustração demonstrativa do passo-a-passo da técnica. O vídeo foi elaborado de acordo com os principais procedimentos de limpeza recomendados pela Organização Mundial da Saúde (OMS). Para a OMS a higienização das mãos é vista como "a medida de higiene mais importante na prevenção da propagação da infecção". Atualmente, a COVID-19 é definida como síndrome respiratória aguda grave, sendo alarmante devido ao seu aumento de número de casos e a disseminação global. De acordo com a OMS, dentra as estratégias recomendadas para prevenir a contaminação está a higienização correta das mãos (com água e sabão ou álcool gel), a utilização de máscara em ambientes públicos e o isolamento social (NOORIMOTLAGH, 2020).

A pandemia provocada pelo novo coronavírus, fez a prática de higienização das mãos se tornar ainda mais indispensável nas ações de prevenção de doenças infecciosas, e paralelamente, diversas outras doenças gastrointestinais agudas, pneumonias e gripes tiveram reduções nas taxas de infecções, evidenciando a relevância desta prática para a saúde pública (CROSBY, 2020).

Com estas propostas de Atividades Didáticas Teóricas e Práticas (ADTP) no ensino/aprendizagem de microbiologia buscou-se envolver conceitos, fundamentações e protocolos que pudessem vir a contribuir para ampliação das possibilidades de aprendizagem dos estudantes envolvidos, permitindo-lhes melhor identificação de microrganismos. Para além disto, buscou-se sensibilizá-los para a adoção dos cuidados necessários para uma melhor profilaxia de doenças causadas por microrganismos e, assim contribuir para uma promoção da educação em saúde pública, tanto de forma individualizada como de forma mais coletiva. Foi considerado que os estudantes agiriam como multiplicadores, conduzindo os conceitos aprendidos e transferindo-os para os familiares e demais pessoas do seu convívio. Sem desconsiderar que, a médio e longo prazo, tais conteúdos serão repassados em contextos de salas de aulas no exercício futuro da docência, para o qual todos os envolvidos neste estudo estão sendo preparados.

Devido ao formato versátil das propostas apresentadas nesse projeto, observou-se que as mesmas poderão ser adaptadas pelos professores, tanto para o ensino superior como para o ensino 
DOI: $10.46667 /$ renbio.v14i2.508

básico, não se restringindo ao ensino de microbiologia, podendo esta vir a ser devidamente adaptada às outras subáreas da biologia, a depender da ADTP que se quer promover. Em Citologia, por exemplo, poderá ser mantida a ADTP 1 e alterar as demais ADTPs propondo, por exemplo, a preparação de lâminas com cortes e coloração de material biológico. Em botânica, a visualização de célula vegetal 3D, caracterização e observação da estrutura de um cloroplasto e de pigmentos vegetais como a clorofila seriam outras sugestões de ADTPs.

Além disso, unir o conteúdo teórico com a realização da prática pela exposição de vídeo e a disponibilização do link de acesso para o laboratório virtual, como feito nesse circuito de microbiologia, é de notória importância uma vez que explora diversos recursos didáticos, aumentando as chances do conteúdo ser aprendido, levando em consideração que a pluralidade metodológica oferece uma maior retenção de informações. Quando avaliamos a subjetividade dos alunos em relação aos conteúdos e atividades do circuito, os comentários dos alunos participantes reforçaram que as propostas aplicadas nas atividades foram satisfatórias:

"Eu gostei de todos os assuntos do circuito. Mas, coloração de Gram foi algo novo para mim" (ALUNO 1); "No geral todos os assuntos foram bem produtivos" (Aluno 2); "Gostei muito da prática de Gram. Bem explicado e direto. O vídeo complementar super bom também”(Aluno 3).

Destacamos ainda alguns comentários dos voluntários participantes do COM sobre a prática da ADTP 2 onde destacam suas percepções e entendimentos sobre a importância de se fazer o procedimento correto de higienizar as mãos.

"Gostei muito da prática da lavagem correta das mãos", "Achei interessante a prática de lavagem das mãos, que nesse período de pandemia é de suma importância", "Achei produtiva a prática de lavagem das mãos pois, fala de forma significativa sobre a importância de lavar e higienizar as mãos".

Após a realização das atividades do COM, foi disponibilizado aos participantes um questionário online com perguntas referentes ao nível de satisfação dos participantes (Figura 02). Em relação ao nível de satisfação, dos 31 alunos que responderam ao questionário, 23 (76,8\%) se mostraram satisfeitos com o COM e 8 alunos $(23,92 \%)$ se mostraram insatisfeitos diante da abordagem. 
DOI: $10.46667 /$ renbio.v 14 i 2.508

Figura 2: Nível de satisfação ou insatisfação dos estudantes voluntários quanto o Circuito Online de Microbiologia (COM)

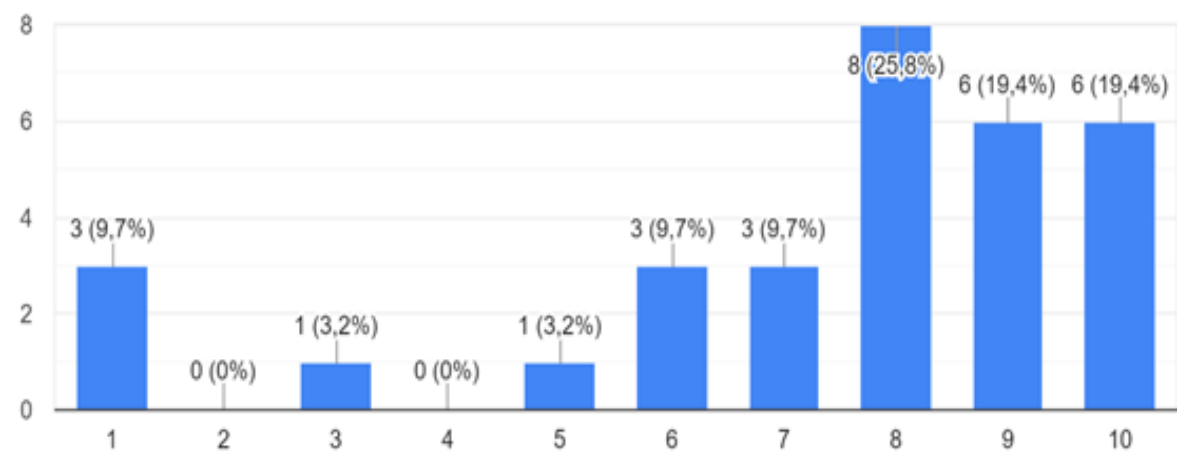

Fonte: Dos próprios autores

Destacou-se alguns depoimentos sugestivos de estudantes que se consideram insatisfeitos com o COM, tais como: “...continuem buscando trazer os conteúdos de uma maneira dinâmica e de fácil compreensão, os vídeos e materiais não são extensos e isso facilita o aprendizado." "Que o grupo aprimore mais os vídeos (pois são bons e podem ficar muito melhor) e também façam mais vídeos para disponibilizar em um site, por exemplo."

Consideramos fundamental a contribuição dos participantes no sentido de melhorar as ADTPs realizadas, já que se percebeu que, mesmo aqueles que se consideram insatisfeitos, trouxeram em suas falas, argumentos positivos, demonstrando o interesse na ampliação do número de atividades e na criação de um site específico de armazenamento dos vídeos para consultas posteriores.

Merazzi (2008) destaca que é de grande importância a percepção da avaliação do aluno quanto ao conteúdo e a forma como ele pode ser trabalhado, proporcionando um melhor ciclo de interatividade e motivando o educando a querer aprender, despertando a importância e utilização desse conteúdo em seu cotidiano. Nesse sentido, conhecer a satisfação dos alunos bem como as sugestões dadas por eles possibilita um melhor planejamento e aperfeiçoamento para os próximos eventos.

Reconhecemos a importância da realização das práticas de microbiologia de forma presencial em laboratórios para esta finalidade. Entretanto, percebe-se que em se tratando de ensino remoto, faz-se necessário a utilização de ferramentas didáticas digitais para que os alunos do curso de licenciatura em ciências biológicas possam observar a realização de práticas importantes para a efetivação do processo de aprendizagem, tornando o ensino mais eficiente nesse formato emergencial de aulas remotas (MASCARENHAS et al., 2016).

\section{Considerações finais}


DOI: $10.46667 /$ renbio.v 14 i 2.508

A proposta implementada pelo COM permitiu que, em um momento de ensino remoto emergencial, fosse possível explorar didaticamente alguns dos principais conceitos sobre os microorganismos, por meio do uso das Tecnologias da Comunicação e Informação, que contribuíram para a realização de práticas online em microbiologia.

Os instrumentos metodológicos utilizados no circuito despertaram bastante interesse nos estudantes, levando em conta que houve um grande participação e interação dos mesmos. Além disso, a proposta do COM proporcionou a construção de conhecimentos de grande importância na temática de microbiologia. Além de tudo, a proposta deu importante contribuição para a formação dos futuros docentes, desafiando estes na criação de propostas que fogem do ensino tradicional e que cooperam para uma efetiva aprendizagem dos seus educandos.

\section{Referências}

AZEVEDO, T. M.; SODRÉ, L. Conhecimento de estudantes da educação básica sobre bactérias: saber científico e concepções alternativas. Revista de Educação, Ciências e Matemática. v. 4, n. 2. 2014.

BRASIL. Ministério da Educação. Conselho Nacional de Educação. Conselho Pleno. Parecer CNE/CP n' 5/2020. Brasília, 2020. Disponível em: http://portal.mec.gov.br/index.php?option=com docman\&view=download\&alias=147041pcp009 - 20\&category_slug=junho - 2020-pdf\&Itemid=30192 . Acesso em: 12 set. 2020.

BERNARDI, G.; LEONARDI, A. F.; SILVEIRA, M. S.; FERREIRA, S. A.; GOLDSCHMIDT, A. I. Concepções prévias dos alunos dos anos iniciais sobre microrganismos. Revista Ciências e Ideias. v. 10, n. 01. 2019.

GIL, A. C. Como elaborar projetos de pesquisa. 4.ed. São Paulo: Atlas, 2002.

KAHOOT. What is Kahoot. Disponível em: https://kahoot.com/what-is-kahoot/ Acesso em: 25 de jul. 2021.

MASCARENHAS, M. J. O.; SILVA, V. C.; MARTINS, P. R. P.; FRAGA, E. C.; BARROS, M. C. Estratégias metodológicas para o ensino de genética em escola pública. Pesquisa em Foco. v. 21, n. 2, p. 05-24. São Luís, 2016.

MERAZZI, D. W.; OAIGEN, E. R. Atividades práticas em ciências no cotidiano: valorizando os conhecimentos prévios na educação de jovens e adultos. Experiências em Ensino de Ciências, Cuiabá, v. 3, n. 1, p. 65-74, 2008.

NOORIMOTLAGH, Zahra et al. A systematic review of emerging human coronavirus (SARSCoV-2) outbreak: focus on disinfection methods, environmental survival, and control and prevention strategies. Environmental Science and Pollution Research, v.1, n.1, p. 1-15, 2020 
DOI: $10.46667 /$ renbio.v 14 i 2.508

OMS. OPAS Brasil. Folha informativa - COVID-9 (doença causada pelo novo coronavírus). Disponível em: https://www.paho.org/bra/index.php?option=com_content\&view=arti cle\&id=6101: covid19\&Ite mid=875\#historico Acesso em: 28 ago. 2021.

PRADO, I. A.C.; TEODORO, G. R.; KHPURI, S. Metodologia de ensino de microbiologia para ensino fundamental e médio. ENCONTRO LATINO-AMERICANO DE INICIAÇÃO CIENTÍFICA, 8., ENCONTRO LATINO-AMERICANO DE PÓS-GRADUAÇÃO UNIVERSIDADE DO VALE DO PARAÍBA, 4. Anais...Disponível: http://www.inicepg.univap.br/cd/INIC_2004/trabalhos/inic/pdf/IC2-11.pdf . Acessado em: 20 mar. 2020.

SARMENTO, E. C.; VERAS, D. S.; ANDRADE, M. V. M. Educação ambiental para reduzir impactos no riacho Lamego: ações na escola Arlindo Fernandes de Oliveira, residencial Eugênio Coutinho. Revista Educação Ambiental em Ação, n. 59: p.2667. 2017.

SCHEID, N. M. J.; FERRARI, N.; DELIZOICOV, D. Concepções sobre a natureza da ciência num curso de ciências biológicas: Imagens que dificultam a educação científica. Investigações em Ensino de Ciências. v. 12, n. 02, p. 157-181. 2007.

WANG, A. I. The wear out effect of a game-based student response system. Computers and education. v. 82, p. 217-227, 2015.

Revisão gramatical realizada pelos autores 